\title{
La migración de niños y niñas de Centroamérica hacia los Estados Unidos: amenaza emergente transnacional
}

\section{Central American Children Immigration to the United States: an Emerging Transnational Threat}

\section{A migração de crianças da América Central para os Estados Unidos: nova ameaça transnacional}

\section{Willy Soto-Acosta}

Profesor de la Escuela de Relaciones Internacionales

María Fernanda Morales-Camacho

Estudiante de Licenciatura en Relaciones Internacionales

Universidad Nacional de Costa Rica

Recibido: 20/3/2015 -Aceptado: 30/6/2015

http://dx.doi.org/10.15359/tdna.31-58.3

\section{Resumen}

La oleada migratoria de menores que viajan sin compañía hacia los Estados Unidos ha sido catalogada como una crisis humanitaria por Washington y ha llamado la atención sobre las condiciones que enfrentan los niños y las niñas de El Salvador, Honduras y Guatemala; donde factores como la carencia de oportunidades de desarrollo, el incremento de la violencia en los crímenes y motivaciones como la reunificación

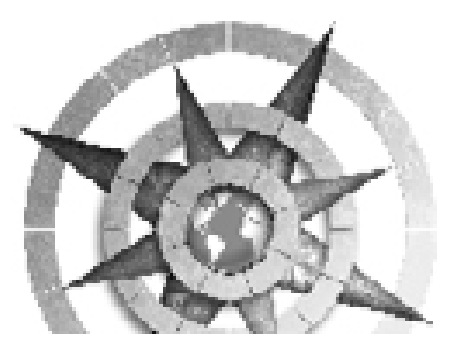

familiar, alentado por las falsas promesas que ofrece el coyotaje sobre las oportunidades de quedarse legalmente en los Estados Unidos y aumenta el éxodo; sin medir los riesgos que enfrentan al realizar el recorrido: asesinato o secuestros para fines de explotación sexual o laboral por parte de los carteles de la droga en su paso por México.

Palabras clave: Migración infantil, niñez latinoamericana en riesgo, crisis humanitaria, Temas de Nuestra America.

\section{Abstract}

The immigration wave of non-accompanied children heading for the United States, has been classified as a humanitarian crisis by Washington 
and, since then, has drawn a closer attention to the living conditions of children from El Salvador, Honduras and Guatemala face. Issues such as the lack of opportunities for development, the increase of violent crimes and hopes for family reunification, along with the coyote's encouraging false promises on hopes for opportunities to legally remain in the United States, are prevailing in this humanitarian crisis. Some perils these children face when making the trip through Mexico are murder, kidnapping for sexual work and labor exploitation by drugs' cartels. Such risks are simply alarming.

Keywords: Migrating children, Latin American children at risk, humanitarian crisis

\section{Resumo}

A onda migratória de crianças desacompanhadas que viajam para os Estados Unidos tem sido classificada como uma crise humanitária por Washington e chamou a atenção para as condições que crianças de El Salvador, Honduras e Guatemala enfrentam. Fatores como a falta de oportunidades para o desenvolvimento, o aumento da violência nos crimes, ou reunião de família, encorajada pelas falsas promessas oferecidas pelo coiote sobre oportunidades de permanecer legalmente nos Estados Unidos, determinou esta crise humanitária. Os riscos que as crianças enfrentam ao fazer a viagem como o assassinato, ou sequestro para operacao com fins sexual ou trabalho escravo por cartéis de drogas em seu caminho através do México, são alarmantes.

Palavras chave: Crianças imigração, infância latino-americana em risco, crise humanitária

\section{Introducción}

La oleada migratoria de menores que viajan sin compañía hacia los Estados Unidos ha sido catalogada como una crisis humanitaria por Washington. Ha llamado la atención las condiciones que enfrentan los niños y las niñas de El Salvador, Honduras y Guatemala. En estos países, factores como la carencia de oportunidades de desarrollo, el incremento de la violencia en los crímenes y motivaciones como la reunificación familiar, alentados por las falsas promesas que ofrece el coyotaje sobre las oportunidades de quedarse legalmente en los Estados Unidos, inciden en estas migraciones. Estas se caracterizan por los riesgos que enfrentan los niños y las niñas al realizar el recorrido: asesinato o secuestros para fines de explotación sexual o laboral por parte de los carteles de la droga en su paso por México.

Este trabajo revisa los antecedentes históricos del fenómeno de la migración de niños/as y adolescentes hacia los

56 La migración de niños y niñas de Centroamérica hacia los 
Estados Unidos hasta llegar al momento actual de esta tragedia humanitaria.

\section{Tres momentos en la historia cen- troamericana reciente}

Es imposible entender las raíces del problema actual de las migraciones de niños/as de la región centroamericana hacia los Estados Unidos sin comprender tres fenómenos relativamente recientes que constituyen antecedentes de este problema:

- Guerras civiles: una época convulsa

Posterior a la Segunda Guerra Mundial, Centroamérica experimentó una estabilidad política y económica relativa, con índices de crecimiento de 5,2 \% (Rojas y Solís, 1988: p.70) y un intercambio regional con tendencia al alza producto de la iniciativa del Mercado Común Centroamericano. Sin embrago, dicha bonanza se vio afectada a mediados de los setenta por la crisis del petróleo, que afectó fuertemente a las economías regionales, dada la evidente pobreza de estos países en materia energética y su clara dependencia externa del hidrocarburo.

Si bien se dio un crecimiento económico importante antes de la crisis del petróleo, la riqueza que obtenían los países centroamericanos no se redistribuía y se concentrada en unos pocos, lo cual profundizó la desigualdad y exclusión existente; misma que se agravó con la contracción económica producto del incremento del precio en el crudo, que incidió en el aumento de la inflación y la deuda de los países de la región; redundando ello en mayores niveles de pobreza.

Sin embargo, aunque el Mercado Común subsistió, no se sostuvo y no logró crear las condiciones permanentes que sostuvieran un nivel competitivo durante el tiempo necesario, y solo benefició a ciertos estratos superiores de la población. El campesinado y la masa trabajadora no sintieron el cambio. La población centroamericana siguió creciendo a una tasa sumamente elevada, especialmente en los estratos de peones rurales y del proletariado, lo que produjo abundancia de mano de obra barata, situación atractiva para las plantaciones agrícolas y empresas transnacionales, pero socialmente peligrosa como tiempo después se ha mostrado (CEPAL citado por Antillón, 1997: p.36).

Al factor socio-económico, se unen aspectos políticos, ideológicos e incluso, culturales que constituyeron el caldo de cultivo para la explosión

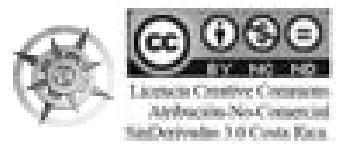


de guerras civiles; tal y como lo explican Rojas y Solís (1988):

La crisis centroamericana responde en gran medida a la falta de equidad económico-social, de participación política y cultural, y de democracia. A estos elementos originarios se han agregado otros de carácter ideológico que han mutado las características del conflicto y sus posibles soluciones, particularmente con la ubicación de la crisis en el contexto Este-Oeste (p.68).

En el nivel político, es posible distinguir sucesos que incidieron para el desarrollo de la crisis; en primer lugar, el ejercicio despótico del poder que incurría a la fuerza ante cualquier intento de cuestionamiento u oposición; lo que provocó "una fuerte inquietud política y una constante inestabilidad" (Rojas y Solís, 1988: p. 70). Por su parte, la marcada desigualdad en las economías que estaban creciendo, provocaba el descontento social y no se evidenciaban esfuerzos para disminuirla por parte de los Gobiernos mediante la ejecución de políticas sociales contra la pobreza, el analfabetismo, las mejoras del bienestar general de los y las centroamericanos/as.

El panorama político y económico de la región excluía a los grupos más vulnerables, quienes empezaron a mostrar su malestar por ser invisibilizados; tal y como lo explica Pérez (citado por Antillón, 1997) al mencionar que "las grandes mayorías no sólo estuvieron excluidas de los beneficios del crecimiento económico, sino también del juego político" (p. 35).

Aunado a lo anterior, se encuentra el aspecto político-ideológico. En un contexto de Guerra Fría, la llegada al poder en Nicaragua del Frente Sandinista de Liberación Nacional en 1979 y la actuación de los grupos revolucionarios en El Salvador (FDR-FMLN), provocó la reacción de los Estados Unidos mediante una política intervencionista basada en el apoyo a los grupos contrarrevolucionarios y a las dictaduras prevalecientes para que no atentaran contra los ideales democráticos de Occidente.

Este fue el caso del apoyo a los "contras" en Nicaragua, bajo una doctrina que respondía a la solución militar como la única posible para asegurar la paz en la región, como medio para mostrarle al mundo, especialmente a la Unión Soviética, que tenían control sobre una zona de influencia inmediata como lo era Centroamérica (estrategia esta que se acentúo con la llegada del presidente Ronald Reagan a la Casa Blanca).

58 La migración de niños y niñas de Centroamérica hacia los 
Los enfrentamientos sociales se convirtieron en guerras civiles y amenazaban con regionalizarse; por lo que la atención del mundo se centró en Centroamérica. En este contexto, sobresalen los esfuerzos de países vecinos, como Venezuela, México y Colombia, por proponer acuerdos que llevaran al establecimiento de la paz.

- El proceso de pacificación: "solución" política sin solución socioeconómica

La firma de Esquipulas II (1987), condujo a la consolidación de la paz en la región y a una eventual democratización de los países afectados por la guerra (Honduras, Nicaragua, El Salvador y Guatemala). La urgencia por cesar los enfrentamientos era tal que los acuerdos de paz se centraron en el aspecto político, pero dejaron de lado las propuestas sociales y económicas en sociedades que quedaron divididas por el resentimiento y la pobreza. Los Estados quedaron destruidos en el nivel de infraestructura y con modelos económicos de base monoexportadora que demostraron que no contribuían al desarrollo socioeconómico de los países. Tal y como lo mencionan Rojas y Solís (1988: p. 77): "El Plan de Paz suscrito por los Presidentes del área centroamericana en la reunión de Guatemala el 7 de agosto de
1987 cambió la dinámica de la crisis regional aún cuando, en un sentido estricto, 'todo cambió sin cambiar nada". Así también lo exponen Netzahualcoyotzi y Furlong (2013):

La reactivación económica esperada como fruto de la paz, no sobrevino. Los acuerdos de Esquipulas I y II (desde 1986) no contenían un nuevo modelo de desarrollo, mucho menos de crecimiento, y la prioridad fue la seguridad y las fronteras como se planteó en el Marco de Seguridad en Centroamérica (Art.10 y 11 del Tratado Marco de Seguridad Democrática en Centroamérica; en Villalta, 2007: 8), antecedente del Plan Colombia y el Plan Mérida (p. 77).

Lo anterior se evidencia en la estructura del Acuerdo de Paz, el cual abordaba tópicos como: reconciliación nacional, exhortación al cese de hostilidades, democratización, elecciones libres, cese de ayuda a fuerzas irregulares o a los movimientos insurgentes, negociaciones en materia de seguridad. También incluía aspectos como verificación, control y limitación de armamento, refugiados y desplazados, cooperación, democracia y libertad para la paz y el desarrollo, verificación y seguimiento internacional y un calendario de ejecución de compromisos. 
Tales puntos se enfocan en la solución política, pero no profundizan en los lineamientos económicos que la región debe seguir para recuperarse de la crisis. Crisis que no es producto de la guerra, sino un problema estructural histórico del Istmo (Rojas y Solís, 1988: p. 143).

- Centroamérica después de Esquipulas II

La ejecución de los Acuerdos de Esquipulas II lograron no solo el cese de fuego, sino el impulso al inicio y consolidación del proceso democrático en la región; constituyéndose, de esta forma, en un proceso de pacificación innovador por promover la acepción positiva de la paz y que sería tomado como referencia en otros procesos de paz en el mundo (Montobbio, 2012: p. 4). Prueba de ello fue la celebración de elecciones democráticas en 1990 en Nicaragua.

La estabilización política incidió en la revitalización económica de la región, que experimentó una tasa de crecimiento del 4,2\% para el periodo 1990-1999 frente al 1,9\% correspondiente a los años 1980. 1990 (León, 2001) y en el fortalecimiento del proceso de integración regional con la creación del Sistema de Integración Centroamericana (SICA) (1991). También incidió positivamente en el incremento en los flujos de comercio intrarregional, constituyendo el proceso de integración económica de la región como uno de los más exitosos en el mundo.

No obstante, la violencia política del periodo de guerras pasó al ámbito privado a cargo del crimen organizado. Se profundiza una violencia estructural y cultural manifiesta en la pobreza y en la aguda desigualdad existente, así como en la segregación de grupos como los indígenas de los proyectos de desarrollo de los Estados. Tal y como lo explica Montobbio (2002):

(...) Bien es cierto que gracias a ella [la paz] no se da en Centroamérica el recursos a la violencia directa como vía de acción política; pero no lo es menos que el principal problema que afronta hoy es el recurso a ésta como vía de acción colectiva por parte del crimen organizado y el cuestionamiento al monopolio del uso de la fuerza por parte del Estado que ello conlleva (p. 5).

Asimismo, en el proceso de democratización regional se encuentra el desafío de no sólo realizar elecciones periódicas sino de promover la participación ciudadana y social brindando espacios para que la sociedad civil se pronuncie sobre el camino que desean para sus países. 
Las migraciones tradicionales de Centroamérica hacia EE.UU.

Según Casasfranco (2002: p. 78) la dinámica migratoria en la región centroamericana fue más notoria a partir de la década de los ochenta y producto de la crisis política en la que estaba sumergida la región. Antes de esa fecha, los desplazamientos obedecían a procesos de colonización agrícola y se caracterizaban por su dispersión en diferentes regiones a lo interno del país. Incluso, en el contexto de la II Guerra Mundial, Centroamérica fue una región receptora de inmigrantes provenientes de Europa y Asia.

Para el caso de México y de Centroamérica, los desplazamientos hacia la frontera norte no fueron regulares, pues están y estuvieron más vinculados por efectos coyunturales (crisis), demanda temporal en el sector agrícola, y no correspondían a todos los países del continente. Además, los desplazamientos se incrementaron a partir de los periodos de guerra o entreguerras, pero con mayor énfasis en los años ochenta del siglo pasado (Netzahualcoyotzi y Furlong, 2002: p. 74)

De esta forma, en la década de los ochenta las guerras civiles incidieron en un desplazamiento cercano a los dos millones de personas que provenían de El Salvador, Guatemala y Nicaragua y se dirigían a otras zonas de su propio país no afectadas por los enfrentamientos, a países vecinos o a destinos extrarregionales. Ejemplo de ello fue la oleada migratoria de ciudadanos/as salvadoreños/as hacia los Estados Unidos: para inicios de la década del noventa, en el país norteamericano residían cerca de un millón de salvadoreños/as; muchos de ellos expulsados por la violencia en su tierra natal (ver tabla 1).

De acuerdo con la Tabla 1, hay un descenso en la población salvadoreña residente ilegal en los Estados Unidos en la década de los noventas respecto al año 2000 , producto de las prerrogativas brindadas por el gobierno estadounidense como el estatus de protección temporal, a principios de los años noventas. Más tarde, se deberá a leyes como NACARA (Nicaraguan Adjustment and Central American Relief Act). No obstante, se evidencia una tendencia al alza en la llegada de hondureños y guatemaltecos a los Estados Unidos en condiciones irregulares; con crecimientos relativos de $96 \%$ y $26 \%$, respectivamente (Homeland Security, 2000: p. 9).

Con el proceso de pacificación en Centroamérica inicia el retorno de 


\section{Tabla 1}

\section{Estimado de población (en miles) residente no autorizada en los Estados Unidos, Top 15 países: 1990-2000}

\begin{tabular}{|c|c|c|c|c|c|}
\hline \multirow{2}{*}{$\begin{array}{l}\text { Cotrity } \\
\text { of enign }\end{array}$} & \multicolumn{2}{|c|}{ Etaredperpititn } & \multirow{2}{*}{$\begin{array}{l}\text { Grout } \\
1390,000 \\
9+0-4\end{array}$} & \multicolumn{2}{|c|}{ Pexcetstetai icplas } \\
\hline & 2000 & 190 & & 2600 & $\begin{array}{r}1990 \\
6\end{array}$ \\
\hline All countries & $7.0 \%$ & 5.406 & 3.500 & 106045 & 109004 \\
\hline Mesices & 4806 & 2,000 & 2,788 & os $\mathrm{N}$ & 3833 \\
\hline E sulvator & 119 & 198 & -100 & $2.7 \%$ & 3.54 \\
\hline Cuabrala & 144 & $1 \mathrm{ts}$ & 28 & $2.1 \%$ & 3.46 \\
\hline Colvatbia & 141 & $\$ 1$ & 91 & $2.0 \%$ & $1.4 \%$ \\
\hline Headerat" & 138 & 42 & \$4 & $2.0 \%$ & 1.24 \\
\hline Chins & Its & 20 & 4) & $1.0 \%$ & $20 \%$ \\
\hline Ecuadot & 108 & 97 & 71 & $13 \%$ & 1.04 \\
\hline Deninican Repubsit & 91 & 40 & 4) & 134 & 1.346 \\
\hline Pilispites & 85 & 20 & 14 & $12 \%$ & 200 \\
\hline Erasil & 77 & $20^{\circ}$ & 58 & $1.1 \%$ & 0.64 \\
\hline Hait & 76 & 07 & $s$ & $11 \%$ & 196 \\
\hline Let. & 70 & 28 & 4) & $10 \%$ & 2546 \\
\hline $\mathrm{mm}$ & of & 27 & 94 & 0.94 & $0.8 \%$ \\
\hline Kenea & is & 24 & 9! & 034 & $07 \%$ \\
\hline Caniti. & 47 & $2 \geqslant$ & 22 & $0.7 \%$ & 0.74 \\
\hline All celtr cecathet & 795 & 397 & 249 & 11206 & 1535 \\
\hline
\end{tabular}

Fuente: Homeland Security. (2000). Estimates of the Unathorized Immigrant Population Residing in the United States: 1990 to 2000.

migrantes a sus tierras de origen, especialmente de salvadoreños/ as y guatemaltecos/as. No obstante, la persistencia de condiciones económicas adversas y la creciente vulnerabilidad ante fenómenos ambientales como huracanes y terremotos, provoca una nueva oleada de migrantes, esta vez de naturaleza económico. Por ejemplo, el impacto del Huracán Mitch en la región produjo un desplazamiento de 22465 personas hondureñas y una oleada migratoria de nicaragüenses hacia Costa Rica, mientas que el terremoto en El Salvador, en 2001, produjo un importante desplazamiento también. (Casafranco, 2002).

Surgen entonces dos lógicas de desplazamiento: una al sur, especialmente de nicaragüenses hacia Costa Rica y otra al norte, específicamente ciudadanos de Guatemala, Honduras y el Salvador hacia México, pero que tienen como principal destino

62 La migración de niños y niñas de Centroamérica hacia los 
los Estados Unidos, en búsqueda del sueño americano.

Actualmente, y de acuerdo con Where, We're From, para el año 2010 se contabilizaban un total 2 664852 migrantes provenientes de Centroamérica en los Estados Unidos. Dentro de esta cifra sobre sale
El Salvador con 1116420 migrantes residiendo en este país, seguido por Guatemala con 753720 y Honduras con 469 202. Estos tres países representan más del $50 \%$ de las personas centroamericanas en esa nación (ver gráfico 1):

\section{Gráfico 1}

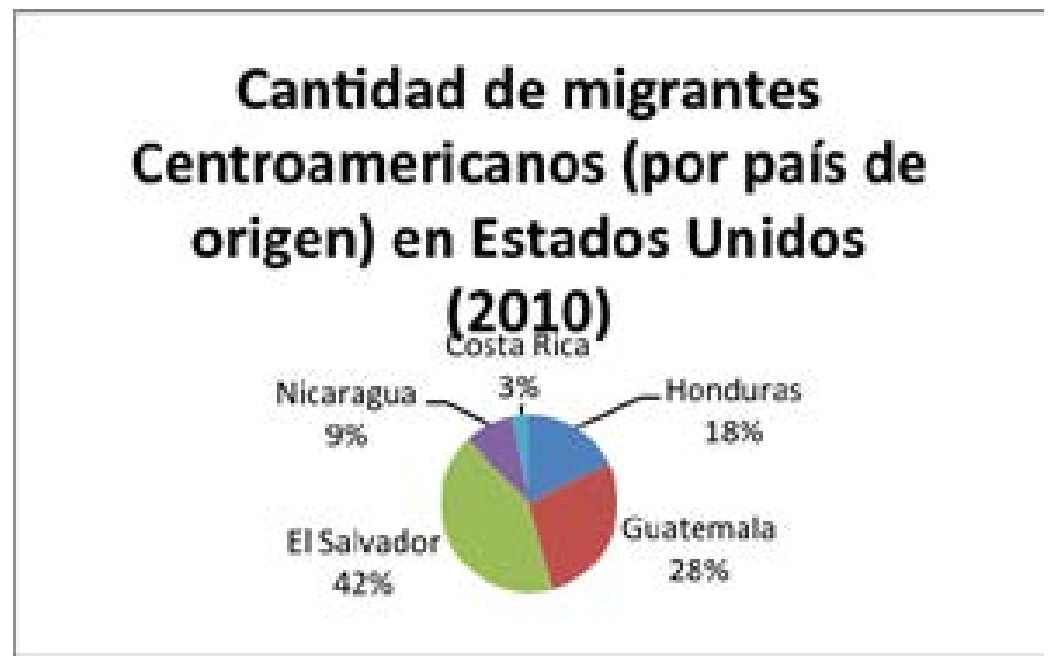

Fuente: Elaboración propia a partir de Organización Internacional para las Migraciones (2014).

Amenazas emergentes en Centroamérica: narcotráfico, maras, crimen organizado, vulnerabilidad y fenómenos ambientales

El proceso de Esquipulas II cerró una etapa convulsa en la región, caracterizada por la amenaza militar a la estabilidad política del istmo. Sin embargo, la región enfrenta nuevos flagelos (algunas veces formas transmutadas de los anteriores). Estos problemas no se limitan a un área geográfica específica, por lo que son más complejos de combatir y obedecen a las lógicas de transnacionalización propias de una sociedad 
más conectada a raíz de los avances en las telecomunicaciones y mucho más porosa, en lo que Beck ha descrito como la sociedad del riesgo (Beck, 2002).

Estos nuevos desafíos se pueden agrupar en grandes fenómenos: el crimen organizado, la amenaza de las maras, la expansión de las redes de narcotráfico y la vulnerabilidad ambiental. Estos factores inciden en la decisión de los y las centroamericanos/as para buscar lugares más seguros y que brinden mayores y mejores oportunidades.

- Crimen organizado y la expansión de las redes de narcotráfico

La región se enfrenta a la profesionalización y a una mayor penetración de las estructuras del crimen organizado en el aparato estatal. Tal y como lo expone el informe Seguridad y Crimen Organizado Transnacional en Centroamérica (2011), la región ha pasado de ser una simple zona de paso, estilo bodega, de narcóticos y paso obligatorio de armas, personas, flora, fauna, capitales ilegítimos, a una zona en la que se han institucionalizado las redes criminales impactando las sociedades centroamericanas con mayores niveles de violencia, con en el aumento en el consumo de drogas y el fortalecimiento de organizacionescomo las pandillas. Tal y como lo explica el estudio:

Durante más de tres décadas, Centroamérica fue utilizada como un puente "natural" para el flujo de las actividades del crimen organizado. En ese contexto, los países de la región, también, fueron sitio apropiado para el almacenamiento de los bienes que ilícitamente se comerciaban. El presente estudio, parte de la hipótesis que la imagen de "puente", "corredor", o "bodega", ya no es suficiente para expresar el desarrollo del crimen organizado en Centroamérica de los últimos años. Esto quiere decir que las actividades del crimen organizado han evolucionado de manera tal que rebasan la idea de que la región es un "puente" o una "bodega". Esta situación va acrecentándose dado que es una zona donde prevalece la falta de oportunidades económicas, y la incapacidad para reducir las desigualdades y la pobreza (Egendoff y Stein, 2011: p. 30-31).

\section{- La amenaza de las maras}

La consolidación de las pandillas más poderosas en Centroamérica (Pandilla de la Calle 18 y la Mara Salvatrucha) así como su acercamiento con el crimen organizado y el nivel de violencia con el que arremeten con la población civil, 
especialmente grupos de mayor vulnerabilidad, como mujeres y niñez, han ocasionado la ejecución de políticas de mano dura por parte de diferentes gobiernos. "Esto ha incidido en la transnacionalización de dichas organizaciones a México, Estados Unidos, incluso Europa" (Comisión Española de Ayuda al Refugiado, 2013: p. 22).

\section{- La vulnerabilidad ambiental}

El cambio climático constituye una de las principales amenazas a la seguridad humana de la región. La fuerte dependencia de las economías de la región de hidrocarburos y los inadecuados usos de la tierra y el agua, suponen desafíos para estos países y sus gobiernos. Temas como la seguridad alimentaria y la gestión de las ciudades costeras ante el inminente incremento del nivel del mar también se han posicionado en la agenda de las políticas públicas centroamericana. Esta región junto con México y República Dominicana verán afectados cerca de un millón de kilómetros cuadrados, lo cual impactará a los ecosistemas y la biodiversidad. Prueba de la vulnerabilidad regional es que en el índice global de riesgo de cambio climático 1990-2008, calculado para 176 países, Honduras ocupó el puesto tercero y Nicaragua el quinto (Meléndez, 2014).

\section{Las migraciones de nueva genera- ción: niños y niñas migrando solos}

De acuerdo con el Reporte International Migration, Children and Adolescents Population Dynamics (2013) de la UNESCO, el $16 \%$ de la población total migrante es joven (menor de 20 años), lo que equivale a 33 millones de personas. De esta cifra, 11 millones oscilan entre las edades de 15 a19 años, mientras que nueve millones cuentan entre 10 y 14 años y aproximadamente siete millones entre los 5 a los 9 años de edad.

El continente americano representa el $10 \%$ de la totalidad de las migraciones mundiales de jóvenes. Asimismo en la región de América Latina y el Caribe las dinámicas migratorias por género han cambiado, al existir 95 mujeres migrantes por cada 100 hombres migrantes menores de 20 años; mientras que para el año 2000 migraban 96 hombres por cada 100 mujeres migrantes. 


\section{Gráfico 2}

Proporción de niños y adolescentes migrantes respecto al total de la población migrante (por región)

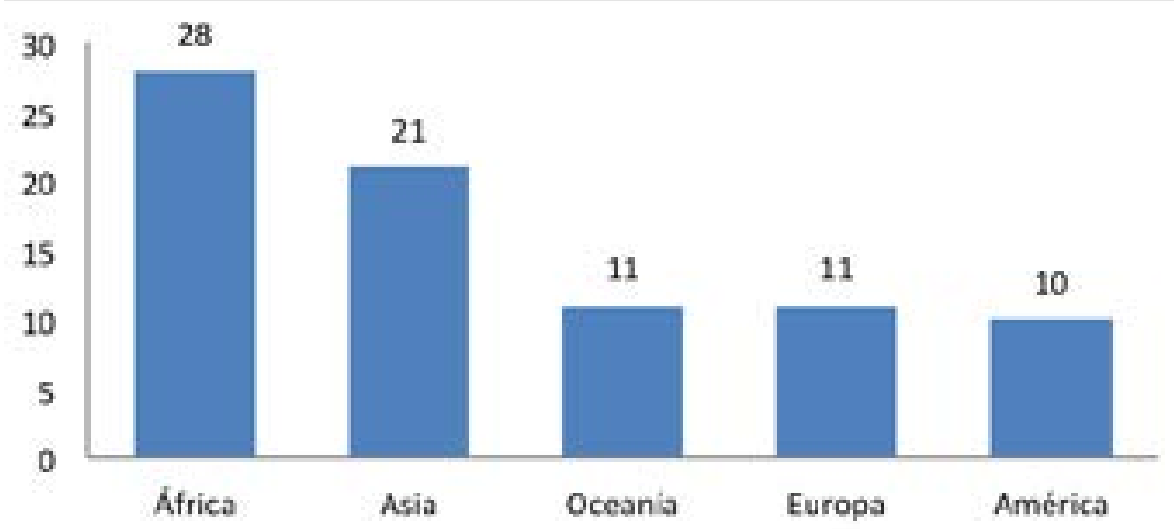

Fuente: UNESCO (2013).

Durante el mes de junio del presente año, la guardia fronteriza de los Estados Unidos ha detenido una avalancha de niños/as que viajan sin compañía para llegar a este país, detonando una crisis de carácter humanitario por las dificultades para atenderlos. Se calcula que desde octubre del año 2013 han llegado y han sido detenidos cerca de 52000 menores (AFP, julio 2014) con una edad promedio de 14 años (Kandel, Bruno, Meyer, Seelke, Taft-Morales y Wasem, 2014: p. 2).

Una gran cantidad son enviados a traer por sus progenitores para reunirse en suelo estadounidense y obtener permisos de estadía. Sin embargo, al revisar los datos de la
Policía de Frontera (2014) es posible identificar una tendencia a la alza en las aprehensiones de menores de edad no acompañados en la frontera entre México y Estados Unidos que, para el año 2009 alcanzó las 2000 detenciones y que llegó a aumentar en ocho veces para el año 2014, superando las 16000 detenciones (caso hondureño). Estos datos reflejan que hay tres países centroamericanos en condición de expulsores netos de menores no acompañados: El Salvador, Guatemala y Honduras (Orozco y Yansura, 2014), mostrando una dinámica migratoria que difiere de la experimentada años atrás.

Mientras que para el año fiscal 2009 cerca del $82 \%$ de la totalidad de 
detenciones de menores no acompañados correspondía a mexicanos y sólo un $17 \%$ a centroamericanos, para el año fiscal 2014 la cifra se revirtió, representando un $23 \%$ los menores provenientes de México y un $75 \%$ del total los originarios de América Central (Kandel y otros, 2014: p.3).

Dichas cifras, junto con las condiciones y riesgos a los que se enfrentan las y los menores llamó la atención no solo de Washington, sino de los gobiernos centroamericanos y de organizaciones internacionales como la ONU, con el fin de brindar alternativas a esta problemática.

En primera instancia, es importante destacar las razones por las cuales los y las niños/as inician un viaje desde sus países de origen hasta los Estados Unidos. Según la investigación Comprender la migración centroamericana: la crisis de migrantes menores de edad centroamericanos en contexto (2014), dicha migración está directamente vinculada con los elevados índices de violencia, tal y como se explica a continuación:

Nuestra investigación ha considerado factores varios que contribuyen al aumento en la migración desde Centroamérica y ha encontrado que la violencia es el impulsor más poderoso e inmediato.
Así como en las décadas de 1970 y 1980 , cuando personas abandonaron la región escapando a la violencia, hoy, en el siglo 21, la juventud está siendo perseguida por fuerzas paramilitares, maras, carteles, traficantes de armas y extorsionistas (Orozco y Yansura, 2014: p. 16).

Al factor violencia debe sumársele las escazas oportunidades para mejorar la calidad de vida a la que se enfrentan los y las jóvenes en las economías centroamericanas, especialmente en los principales países expulsores, Honduras, Guatemala y El Salvador, donde el incremento de la informalidad, reducidas oportunidades de trabajo calificado y los problemas para tener acceso al sistema educativo, y mantenerse en él, son catalizadores en la decisión de dejar el país. Así lo explican los investigadores: “(...) la pobreza no impulsa la migración, pero sí lo hace la ausencia de desarrollo: cuando existen oportunidades suficientes para una sociedad moderna, emigrar se convierte en una alternativa atractiva" (Orozco y Yansura, 2014: p. 16)

Así mismo, en un reciente informe del Congreso denominado Unaccompanied Alien Children: Potential Factors Contributing to Recent Immigration (2014) se han determinado dos tipos de causas para la creciente 
oleada migratoria de niños/as y adolescentes provenientes del Triángulo del Norte: los factores push, que son aquellos que originan la salida del país de nacimiento y los factores pull, identificados como los motivadores (originados en el país de destino) para trasladarse a otro lugar (Kandel y otros, 2014: 1), tal como se analiza en el informe. A continuación se presentan factores push y factores pull:

Factores push: el informe analiza dos situaciones condicionantes: primero, el entorno socioeconómico; segundo, el crimen y la violencia. En el caso del entorno socioeconómico se identifican aspectos como un crecimiento lento de las economías y una marcada desigualdad social. Aunado a ello está el impacto de fenómenos naturales en las economías locales, cuya mayoría son de base agrícola. Por su parte, los elevados índices de violencia en estos países, expresados por ejemplo en las alarmantes tasas de homicidio y en la penetración y expansión de las estructuras del crimen organizado (narcotráfico y pandillas como las Maras o M-18), inciden en el incremento de la inseguridad y el temor por conservar la integridad física ante prácticas como la extorsión, el secuestro y el reclutamiento de jóvenes en las organizaciones.

Factores pull: la percepción de mejores oportunidades económicas y educativas, la reunificación familiar y las recientes políticas de inmigración estadounidenses son, probablemente, las causales más importantes para la llegada de inmigrantes, mismas que se consideran más subjetivas si se comparan con las causales push (Kandel y otros, 2014: p. 12). En el caso de la percepción de mejores oportunidades económicas y educativas, ellas corresponde al acceso a mejores fuentes de trabajo por parte de los padres y madres de familia o bien otra parentela; así como la posibilidad de ingresar al sistema público de educación primaria y secundaria frente a la inexistente oportunidad de estudiar en los países expulsores, tal y como lo explican los autores del informe:

Apart from what unaccompanied children cite as pull factors, U.S. labor market conditions likely affect their parents and relatives residing in the United States, which in turn, may play a critical role in the recent surge. Improving employment prospects, for instance, could more readly

68 La migración de niños y niñas de Centroamérica hacia los 
provide parents with the means to afford the expense of their children's migration to this country and lead to greater desire for family reunification as discussed below (Kandel y otros, 2014: p. 3).

Por otra parte, la reunificación familiar se considera como uno de los principales catalizadores de la migración de niños/as y adolescentes (ver Tabla 1); principalmente, ante la mejora en las condiciones de vida de sus padres o familiares que residen en los Estados Unidos y la creciente preocupación por el bienestar de aquellos ante las amenazas percibidas en los países de origen, apoyándose en que la legislación migratoria permitirá la estadía de las personas menores de edad en suelo estadounidense (creencias que se alejan, en muchos casos, de la realidad y son difundidas por las redes criminales

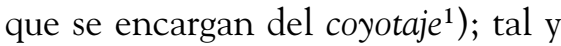
como se explica en informe de Kandel y otros: "The desire for family reunification is also driven by the perception that children who are not

1 Por ejemplo los coyotes indican a los padres de familia que apoyándose en iniciativas como Deferred Action for Childhood Arrivals, en el marco de la reforma migratoria propuesta por la administración Obama, los menores se podrán quedar legalmente en el país. Lo que no mencionan es que DACA cubre sólo a menores de 16 años que han residido continuamente en los Estados Unidos desde el 15 de junio del 2007 (Kandel y otros, 2014: 20). inmediately to their countries can reside with their family members for periods extending several years (...)" (2014: p. 15).

\section{La reacción de Washington y de los gobiernos Centroamericanos}

Sin duda alguna, la llegada masiva de menores a la frontera entre México y los Estados Unidos ha ocasionado una crisis humanitaria que incide en los intereses de la Administración Obama por lograr que se apruebe la reforma migratoria en el Congreso, y ha puesto en apuros a las autoridades estadounidenses que no se encontraban preparadas, ni en el nivel de gestión ni en términos de personal e infraestructura, para mantener en condiciones decentes a los menores migrantes sin compañía.

Según la comparecencia Children Migrating from Central America: Creating a Humanitarian Crisis (2014), del Secretario Asistente del Bureau de Asuntos para el Hemisferio Occidental, Francisco Palmieri, ante el Comité de Asuntos Exteriores, se ha establecido una coordinación con la Agencia Federal de Gestión de Emergencias (FEMA por sus siglas en inglés) para las respuestas rápidas y de corto plazo. Asimismo, se ha procedido a realizar una campaña propagandística en coordinación 
Tabla 2

Principales razones en la migración de niños(as) no acompañados ${ }^{2}$

\begin{tabular}{|c|c|c|c|c|c|}
\hline $\begin{array}{l}\text { Conutry of } \\
\text { Origin }\end{array}$ & $\begin{array}{l}\text { Societal } \\
\text { Violence }\end{array}$ & $\begin{array}{c}\text { Domestic } \\
\text { Abuse }\end{array}$ & $\begin{array}{c}\text { Ecenomic } \\
\text { Deprivation or } \\
\text { Soctial } \\
\text { Excluilen }\end{array}$ & $\begin{array}{c}\text { Family or } \\
\text { Opportumities } \\
\text { in the United } \\
\text { States }\end{array}$ & Other \\
\hline E Sulndor & 668 & $20 x$ & $\pi$ & $80 x$ & $35 x$ \\
\hline Guevemah & 2008 & $23 x$ & 29 & $84 \mathrm{x}$ & 39 \\
\hline Hendurns & $44 x$ & 245 & 211 & $82 x$ & 365 \\
\hline Mexiles & 585 & $17 x$ & $n$ & $\operatorname{sex}$ & 345 \\
\hline Total & $48 x$ & $21 x$ & $16 \mathrm{~s}$ & $31 \%$ & $25 \mathrm{x}$ \\
\hline
\end{tabular}

Fuente: Unaccompanied Alien Children: Potential Factors Contributing To Recent Immigration (2014) tomado de UNHCR, Children on the Run.

con los gobiernos centroamericanos y de México, orientada a desmentir a las redes criminales sobre la certeza de que las personas llegarán sin problemas a los Estados Unidos y evidenciar los peligros que implica un viaje de este tipo. De la misma forma, se pretende desmentir que los niños no serán deportados. Por otra parte, el Gobierno de Estados Unidos se comprometió a facilitar la suma de 9.6 millones de dólares a los gobiernos centroamericanos para capacitar al personal que se encargará de recibir a las personas menores de edad deportadas.

2 Los datos corresponden a la aplicación una encuesta realizada por el Alto Comisionado de las Naciones Unidas para los Refugiados (ACNUR) en el año 2013 aplicada a 400 menores que llegaron sin compañía a los Estados Unidos provenientes de Guatemala, El Salvador y Honduras en el año 2012.
Los presidentes de Guatemala, Honduras y El Salvador se reunieron a finales del mes de julio de 2014 para discutir las medidas ante la crisis migratoria, entre las que se planteó la posibilidad de regularización de un pequeño grupo de jóvenes, especialmente hondureños, mediante la aceptación de asilo (El Financiero, 25/07/2014). Los presidentes centroamericanos solicitaron fondos para tratar, a largo plazo, problemas estructurales de la región como el narcotráfico y la violencia. Incluso se planteó la propuesta de un Plan Centroamérica por parte del mandatario guatemalteco, Otto Pérez Molina, similar al Plan Colombia (El Universal, 25/07/2014). No obstante, el Presidente Obama enfatizó en la deportación masiva de los menores con un estatus irregular ante un Congreso que se negaba a

70 La migración de niños y niñas de Centroamérica hacia los 
aprobar los 1700 millones de dólares solicitados por el Ejecutivo para paliar la crisis.

\section{A modo de conclusión: Centroa- mérica, una región expulsora de menores...}

La detención de niños/as y adolescentes en la zona fronteriza entre México y Estados Unidos ha evidenciado problemas estructurales de las sociedades centroamericanas, específicamente de los países del Triángulo del Norte; a la vez que plantea una renovada estrategia para la región por parte de los Estados Unidos para enfrentar tan suceso.

En primera instancia, las migraciones responden a una interacción de factores push y pull, generados en los países de origen y de destino, respectivamente. En el caso centroamericano, las migraciones correspondientes a la época de los conflictos armados responden a la búsqueda de oportunidades laborales y a la persecución política de la que era víctima la población civil centroamericana. A diferencia de los flujos migratorios actuales, que obedecen a la violencia persistente en la región, en particular a manos de grupos privados (pandillas, crimen organizado), la amenaza que representan ciertos fenómenos ambientales y el deseo de mantener el núcleo familiar unificado.

La migración de menores no acompañados representa una problemática de mayor envergadura dada su vulnerabilidad y la posibilidad de ser víctimas de las organizaciones criminales durante su trayecto, frustrando así la posibilidad de llegar a suelo estadounidense. Además, este suceso representa no solo un reto para los países centroamericanos a título individual, sino para la institucionalidad centroamericana de integración regional al exigir el diseño y ejecución de políticas orientadas a mejorar las condiciones socioeconómicas de la región; así como atacar los problemas de seguridad y criminalidad existentes. En estos aspectos es menester una acción conjunta con los Estados Unidos en calidad de país destino de migrantes y principal mercado de la droga producida en Suramérica y en México.

Finalmente, la migración es un tema político en la medida que el bienestar de estas personas menores de edad se encuentra en función de las decisiones que se tomen en el Congreso estadounidense respecto a la reforma migratoria y cómo se procederá con su tratamiento legal de esas personas en el tanto son deportadas a sus países de origen. En estos 
aspectos la administración Obama no ha logrado importantes avances, debido a la oposición republicana y dada la inminencia del proceso electoral en Estados Unidos

\section{Referencias bibliográficas}

Antillón S., A. (1997). Contadora: isimples buenos oficios o formal mediación? (Pacificación en la crisis centroamericana). Costa Rica: Universidad Nacional

AFP. (02/07/2014). EU vive crisis humanitaria por la "avalancha" de niños migrantes. Revisado: 01/09/2014. Disponible en: http://mexico.cnn.com/ mundo/2014/07/02/eu-vive-una-crisis-humanitaria-por-la-avalancha-de-ninos-migrantes.

Beck, U. (2002). La sociedad del riesgo global. Revisado: 01/09/2014. Disponible en: http://www.um.es/tic/ LIBROS\%20FCI-II/Beck\%20Ulrich $\% 20-\% 20 \mathrm{La} \% 20$ Sociedad $\% 20$ Del\%20Riesgo\%20Global.pdf

Casasfranco R. M. (2002). Las migraciones y los desplazamientos forzados. Retos en Centroamérica y Colombia. Un análisis comparativo e integral desde un enfoque de derechos humanos. Costa Rica: Impresora Obando:

Comisión Española de Ayuda al Refugiado. (2013). Maras en Centroamérica y México. Disponible en: http://cear.es/ wp-content/uploads/2013/10/CENTROAMERICA.-2013.-Maras.pdf.

Egenhoff, T. y Stein, E. (2011). Seguridad y crimen organizado transnacional. Revisado: 01/09/2014. Disponible en: http://www.kas. de/wf/doc/kas_22430-1522-4-30. pdf?110519174059. Revisado: 01/09/2014.

El Financiero. (25/07/2014). "Obama pide a presidentes centroamericanos ayuda para detener migración" En: El Financiero. Recuperable de: http:// www.elfinanciero.com.mx/mundo/ obama-pide-a-presidentes-centroamericanos-ayuda-para-detener-migracion.html.

El Universal. (25/07/2014). "Inicia Obama reunión con presidentes centroamericanos". En: El Mundo. Revisado: 01/09/2014. Recuperable de: http:// www.eluniversal.com.mx/el-mundo/2014/obama-reunion-presidentes-centroamerica-1025827.html

León, J. (2001). Centroamérica y el mundo en los albores del siglo XXI. En: Polí tica y cultura. Revisado: 01/09/2014. Disponible en: http://www.redalyc. org/articulo.oa?id=26701506.

Kandel, W., Bruno, A., Meyer, P., Seelke, C., Taft-Morales, M., Waseem, R., (2014). Unaccompanied Alien Children: Potential Factors Contributing to recent Inmigration. Revisado: 01/09/2014. Disponible en: http:// fas.org/sgp/crs/homesec/R43628.pdf.

Meléndez, J. (2014). Centroamérica, muy vulnerable al cambio climático. En: El País. Revisado: 01/09/2014. Disponible en: http://sociedad.elpais. com/sociedad/2014/06/09/actualidad/1402324929_244265.html.

Montobbio, M. (2012). Esquipulas II +25: Centroamérica en perspectiva. Revisado: 01/09/2014. Disponible en: http://www.cidob.org/es/

72 La migración de niños y niñas de Centroamérica hacia los 
publicaciones/notes_internacionals/ n1_61/esquipulas_ii_25_centroamerica_en_perspectiva.

Netzahualcoyotzi, R. y Furlong, A. (2013). Migración Centroamericana. En: Mesoamérica: Integración regional y geopolítica. Universidad Nacional: Costa Rica.

Orozco, M. y Yansura, J. (Agosto 2014). Comprender la migración centroamericana: la crisis de migrantes menores de edad centroamericanos en contexto. Diálogo Interamericano: Washington.

Palmieri, F. (2014). Children Migrating from Central America: creating a Humanitarian Crisis. Revisado: 01/09/2014. Disponible en: http://docs.house.gov/meetings/ FA/FA0 7/20140625/102418/ HHRG-113-FA07-W state-PalmieriF-20140625.pdf
Rojas, F. y Solís, L. (1988). ¿Súbditos o aliados?: La política exterior de Estados Unidos y Centroamérica. FLACSO: Costa Rica.

UNICEF. (April 2013). International Migration, Children and Adolescents Population Dynamics. Revisado: 01/09/2014. Disponible en: http://www.unicef.org/ socialpolicy/files/Population_Dynamics_and_Migration.pdf 